\title{
Research on Composite Drag Reduction Characteristics of Biomimetic Microstructural Surface
}

\author{
M. H. Zhang†, D. B. Zhang and W. W. Zhuo \\ Shandong University of Science and Technology, Qingdao, Shandong Province, 266590, China \\ †Corresponding Author Email: zmh1999238@163.com
}

(Received September 1, 2020; accepted February 21, 2021)

\begin{abstract}
The objective of this work is to investigate the effect of the bionic microstructure surface on flow field structure of the slab. The motivation behind this study is to investigate the effect of the bionic microstructure parameters including the height and intersection angle of microstructure in order to improve the drag reduction characters. The numerical simulation is performed on the bionic microstructure model of the V-shaped and serrated bionic microstructures using the RNG k- $\varepsilon$ model. The drag reduction rates of two bionic microstructures under different dimensionless sizes are ob13.79 tained. The drag reduction efficiency is up to $8.76 \%$ when the dimensionless height of microstructure $\mathrm{h}^{+}$is at and the intersection angle $\theta=40^{\circ}$ for $\mathrm{V}$-shaped microstructure. In addition, combined with wall temperature control of drag reduction technology, the influence of wall temperature on the drag reduction effect is also analyzed. Compared with the flow field structure of the surface boundary layer of the smooth plate, the wall microstructure divides the surface boundary layer into two parts: the bottom and the tip. The average velocity profile is moved up and the thickness of the linear bottom layer is increased. A large number of "quiet" fluids are gathered at the bottom of the surface boundary layer. In addition, the existence of wall microstructure can weaken the momentum exchange in the boundary layer and restrain the spreading vortex motion of the fluid in the near-wall region. The "secondary vortex pair" on both sides of the tip of the microstructures can effectively limit the lateral pulsation of fluid So as to achieve a good drag reduction effect.
\end{abstract}

Keywords: Bionic microstructure; RNG model; Numerical simulation; Drag reduction.

\section{NOMENCLATURE}

A body surface area

$C_{F} \quad$ average friction coefficient of wall surface

$C_{\mathrm{d}}$ drag coefficient

$F \quad$ resistance on the surface

$G_{b} \quad$ the turbulent kinetic energy terms produced by buoyancy

$G_{k} \quad$ turbulent kinetic energy terms produced by velocity gradient microstructural height

$h^{+} \quad$ dimensionless height of microstructure

$H$ shape factor

$L \quad$ plate length

$s \quad$ microstructural spacing

$s^{+} \quad$ dimensionless spacing of microstructure

$S_{\mathrm{k}} \quad$ user-defined turbulent kinetic energy terms
$S_{\varepsilon} \quad$ turbulent dissipation terms

$U$ freestream velocity

$u_{\tau} \quad$ wall tangential stress velocity

$y$ height from the wall

$y^{+} \quad$ dimensionless distance from the wall

$\delta$ nominal boundary layer thickness

$\delta^{*} \quad$ boundary layer displacement thickness

$\theta \quad$ microstructure tip Angle

$\mu \quad$ dynamic viscosity coefficient

$\rho$ density of the fluid

$\tau_{w} \quad$ tangential wall stress

$\tau^{R} \quad$ Reynolds shear stress

$v \quad$ kinematic viscosity coefficient 


\section{INTRODUCTION}

The surface friction resistance accounts for the largest proportion of the total resistance in the fields of transportation and pipeline transportation. Therefore, reducing surface friction resistance is one of the main ways to improve energy efficiency. Inspired by bionics, Biomimetic microstructures can effectively improve the turbulent boundary layer structure and reduce the friction resistance on the surface of objects. At present, researchers have done a lot of exploratory research on biomimetic microstructure and made a lot of achievements.

The current research is mainly focused on two aspects. On the one hand, by exploring the drag reduction mechanism of the surface microstructures of sharks, dolphins, birds and so on, the corresponding bionic surface structure is established. The work in this area includes: Luo et al. (2014) established the precise digital models of the shark surface microstructure and found that the microgrooves on the shark skin could increase the viscous bottom layer and have a significant role in inhibiting turbulence and reducing wall viscosity resistance. Pu et al. (2016) studied fine structure of shark skin and found that the specific microstructure had a certain influence on the surface wettability and the air layer fixed on the surface of the bionic shark skin played a key role in its biological adhesion resistance. Dou et al. (2012) manufactured the biomimetic surface which simulates fish scales using the coating technology and carried out the comparison experiment between the bionic surface and the smooth surface in the water cave. They found the surface of the fish scales could effectively reduce the friction resistance. Zhao et al. (2012) manufactured the shark skin replicating membrane by vacuum casting and performed drag reduction experiments in the sink. The drag reduction rate is up to $18.6 \%$. Bechert et al. (2000) established the sharp fin - shaped surface arranged in an interlock array imitated the shark skin. This surface can obtain 7.3\% drag reduction effect. Wu et al. (2018) researched the drag-reduction characteristics of the bionic surface with delicate water-trapping microstructures of fish with experimental method. They found that the bionic surface created a rewarding drag-reduction effect at a low speed and the drag-reduction rate significantly displayed a downward trend with the increase in flow speed. Wang et al. (2015) studied the microstructure on the surface of shark, dolphin, shell and turtle was in detail in order to explore the mechanism of the micro structure for antifouling and drag reduction. Gu et al. (2019) analyzed the influence of the jet aperture, the jet angle and the rotational velocity coupled with the jet velocity on the friction torque acting on a jet surface model by using a jet surface drag reduction testing experimental platform. Besides, Domel et al. (2018) had reported a set of denticle structures inspired by the drag-reduction characteristics of the skin of the sharks by the combination of experimental and simulation methods, achieving simultaneous dragreduction effect and lifting generation on the aerofoil, which effectively improved the aerodynamic performance. Song et al. (2017) proposed a new type of non-smooth surface inspired by the shape of barchans dunes and carried out simulations to obtain skin friction reduction characteristics using a realizable $k-\varepsilon$ model. The maximum drag reduction rate of bionic non-smooth surface is up to $33.63 \%$. Wu et al. (2017) observed and measured exceptional morphologies and structures of the scales using a scanning electron microscope and 3-dimensional (3D) microscope. Then, they analyzed the mechanism of the watertrapping effect of these structures through numerical simulations and theoretical calculations.

Feng et al. (2015) attempted to reveal the biologic features responding to skin friction drag reduction by analyzing the surface microstructure of bird feather. The numerical simulation results showed that microvortex induced in the solid-gas interface of bionic surface had the effect of shear stress reduction and the small level of an additional pressure drag resulting from pressure distribution deviation on bird feather like surface. Chen et al. (2013) investigated microstructures of secondary feathers of adult pigeons are by SEM and proposed novel biomimetic herringbone riblets with narrow smooth edge to reduce surface drag based on quantitative analysis of feather structure. Zhang and Bijay (2018) carried out the experiments and computational research for aero engine blade with micro-texture in order to explore the drag reduction performance. The comparison results show that the established drag reduction model of textured blade has better drag reduction performance than that of un-textured blade and verify that the proposed methods to determine the placement position of micro-texture on blade surface and to optimize drag reduction performance of textured blade are satisfied.

The other is to add grooves or riblets on the smooth surface to study the influence of groove shape and size on drag reduction. Walsh (1983) first started the research on the drag reduction of the microstructure plate and found that there is a drag reduction effect when the dimensionless height $\mathrm{h}+\leq 25$ and dimensionless spacing $s+\leq 30$. Luo and Zhou (1993) point out the coherent structure near the wall can affect the generation of turbulent energy and the microstructure shape is very important factor for the improvement of drag reduction rate. Rowley et al. (2002) investigate the resonant instabilities in two dimensional flow past an open cavity using numerical simulations. Predictive criteria for the onset of shear-layer oscillations (from steady flow) and for the transition to wake mode are developed based on linear theory. Gu et al. (2017) numerically simulated the flow field on the surface of inclined microstructures with the Reynolds stress model (RSM). The influence of the flow direction and the inclination angles was investigated. It showed that the viscous resistance decreases significantly when the speed and inclination angle increase. Schrader (2019) explored possibilities of reducing skinfriction drag by the compliant surface which delay the transition from laminar to turbulent flow. Liu et al. (2015) integrated the bionic non-smooth surface into the design of hydrodynamic torque converter and established the concaves model on non-smooth 
surface to conduct the research on the drag reduction and efficiency improvement. Gregory et al. (2013) studied the effect of viscosity, coatings and the interaction between vortices and riblet surfaces in order to provide design guidance when developing novel low drag and self-cleaning surfaces for applications in the medical, marine, and industrial fields. Mariotti et al. $(2014,2015)$ investigated a plane asymmetric diffuser with contoured cavities and found that a favourable effect of the cavities in reducing the momentum losses near the wall. Raayai-Ardakani and McKinley (2017) explored the effects of periodic sinusoidal riblet surfaces aligned in the flow direction on the evolution of a laminar boundary layer flow. They found that in the laminar boundary layer regime the riblets are able to retard the viscous flow inside the grooves creating a cushion of stagnant fluid which the high speed fluid above can partially slide over, thus reducing the shear stress inside the grooves and the total integrated viscous drag force on the plate. Promode et al. (2014) gave a minimal self-regulation model for wall turbulence regeneration in the transitional regime laterally coupled which restricted to prebreakdown durations and to a plane close and parallel to the wall, correctly reproduces many experimentally observed spatiotemporal organizations of vorticity in both laminar to turbulence transitioning and very low Reynolds number but turbulent regions. Fu et al. (2017) discussed different marine drag reduction technologies and analyzed experimental parameters include riblet geometry, continuous and segmented configurations, fluid velocity (laminar and turbulent flow), fluid viscosity (water, oil and gas), and wettability in order to contribute to a better understanding of marine drag reduction and offer new perspectives to improve the current evaluation criteria of riblet drag reduction. Mariotti et al. (2017, 2019) assessed the effectiveness of properly contoured transverse grooves in delaying the flow separation occurring on a two-dimensional boattailed bluff body through numerical simulations. They found that the introduction of the grooves leads to a regularization of the vortex shedding downstream of the body.

At present, most of the researches on the drag reduction effects of the bionic microstructure surface are based on the condition of a single bionic microstructure surface. The effects of the sizes parameters of bionic microstructures on drag reduction are not given. In addition, there are few studies on the combination of microstructural surface with other drag reduction techniques. As part of efforts to fill the knowledge gap, the model of the Vshaped and serrated bionic microstructures are established. The numerical simulation is carried out using the RNG k- $\varepsilon$ model. Research has focused on the following areas: (1) Effects of parameters of microstructures on drag reduction; (2) Effect of wall temperature on surface boundary layer of microstructures; (3) the internal velocity, vorticity, turbulence parameter, shear stress and flow evolution state of the microstructure were deeply explored from the boundary layer perspective.
The remainder of the paper is organized as follows: Section 2 briefly reviews the establishment of biomimetic microstructure model and found mentals of numerical simulation. In Sections 3, the drag reduction effects of the different shape snd size of microstructures are presented and discussed. In Sections 4, the mechanism of microstructure drag reduction is discussed. Finally, the findings of the present study are summarised.

\section{FUNDAMENTALS OF NUMERICAL SIMULATION}

\subsection{Establishment of biomimetic microstructure model}

In this paper, two different types of bionic microstructure surface will be designed, V-shaped and serrated, for turbulent drag reduction analysis. Figure 1 describes the two types of microstructure shapes, where $h$ is the microstructure height, $s$ is the microstructure spacing, and $\theta$ is the tip angle of the microstructure.

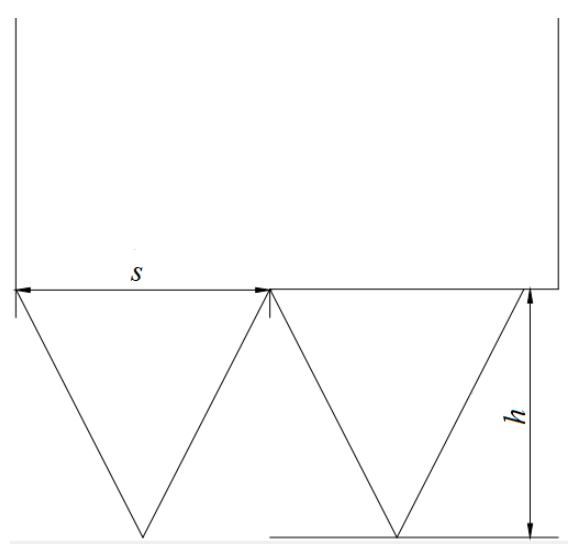

(a)

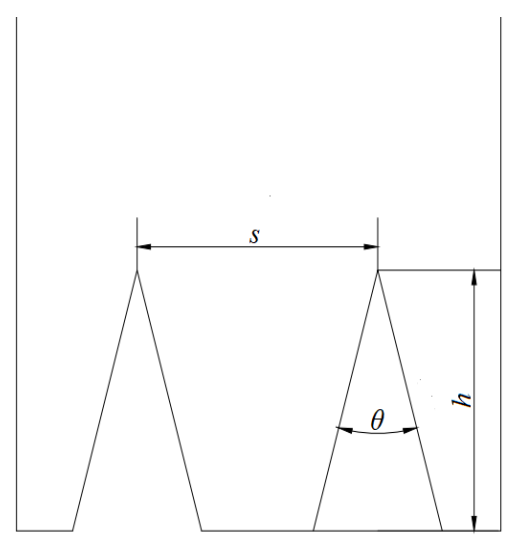

(b)

Fig. 1. Microstructure shapes (a) V-shaped microstructure, (b) sawtooth microstructures.

The dimensionless height $h^{+}$and the dimensionless distance $s^{+}$of the microstructures are defined as:

$$
h^{+}=\frac{h U}{v} \times \sqrt{\frac{C_{F}}{2}}=\frac{h u_{\tau}}{v}
$$


M. H. Zhang et al. / JAFM, Vol. 14, No. 5, pp. 1337-1350, 2021.

$s^{+}=\frac{s U}{v} \times \sqrt{\frac{C_{F}}{2}}=\frac{s u_{\tau}}{v}$

Where, $U$ is the freestream velocity, $v$ is the kinematic viscosity coefficient, $h$ is the height of the bionic microstructure surface, $s$ is the distance between adjacent microstructures, $C_{\mathrm{F}}$ is average friction coefficient of wall surface, $u_{\tau}$ is the wall tangential stress velocity, can be expressed as follows:

$u_{\tau}=\sqrt{\frac{\tau_{w}}{\rho}}$

Where, $\rho$ is density of the fluid, $\tau_{w}$ is tangential wall stress.

According to the empirical formula (Fox et al. 2011):

$C_{F}=\frac{\tau_{w}}{\frac{1}{2} \rho U^{2}}=\frac{0.0594}{R_{e}^{1 / 5}}$

$\tau_{w}$ can be obtained as

$\tau_{\mathrm{w}}=0.0297 \rho U^{2} R_{e}^{-\frac{1}{5}}$

Where, Re is Reynolds number.

By solving Eq. (3) and Eq. (4), $u_{\tau}$ can be obtained as

$u_{\tau}=0.172 U \operatorname{Re}^{-\frac{1}{10}}$

By solving Eq. (1), Eq. (2) and Eq. (5), the dimensionless height and spacing of the microstructures can be estimated as

$h^{+}=\frac{0.172 h U R e^{-\frac{1}{10}}}{v}$

$s^{+}=\frac{0.172 s U R e^{-\frac{1}{10}}}{v}$

The height $h$ of the bionic microstructure surface and the distance $s$ between adjacent microstructures are important factors that affect the drag reduction effects of the bionic microstructure surface. In order to calculate the value of $h$ and $s$, let $U=5.5 \mathrm{~m} / \mathrm{s}$, $\operatorname{Re}=7.53 \times 10^{5}, \rho=1.226 \mathrm{~kg} / \mathrm{m}^{3}, \quad v=1.46 \times 10^{-5} \mathrm{~m}^{2} / \mathrm{s}$. According to Walsh et al.'s conclusion of experimental study on drag reduction effect of micro-structure, the surface of micro-structure has good drag reduction effect at dimensionless height and spacing $h^{+} \approx s^{+} \approx 15$ (Walsh 1983). Therefore, the bionic microstructures will be designed using this conclusion in this paper. Substitute dimensionless height and spacing $h^{+} \approx$ $s^{+} \approx 15$ into Eq. (6) and Eq. (7) in order to obtain the height $h$ of the bionic microstructure surface and the distance $s$ between adjacent microstructures $h=0.9 \mathrm{~mm}, s=0.9 \mathrm{~mm}$. The two types of bionic microstructure size information are shown in Tab.1.

In order to ensure that the flow is in a completely
Table 1 Bionic microstructure size information.

\begin{tabular}{|c|c|c|c|}
\hline Groove Types & Spacing $s$ & Height h & Angle $\theta$ \\
\hline V-shaped & $0.9 \mathrm{~mm}$ & $0.9 \mathrm{~mm}$ & \\
\hline Serrated & $0.9 \mathrm{~mm}$ & $0.9 \mathrm{~mm}$ & $30^{\circ}$ \\
\hline
\end{tabular}

turbulent state and the upper and lower surfaces of the microstructure calculation domain do not interfere with each other, let the total length of the calculation domain $L=2000 \mathrm{~mm}$, the total height of the calculation domain $500 \mathrm{~mm}$ and the width of the span is $1.8 \mathrm{~mm}$, that is twice the height of two microstructures. The bionic microstructure and smooth flat computing domain are shown in Fig. 2.

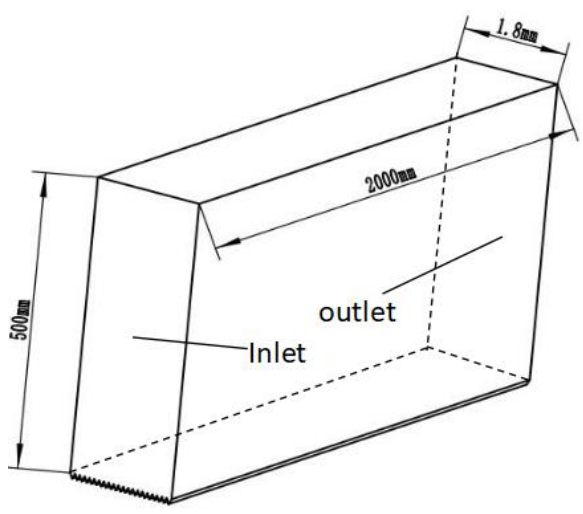

Fig. 2. Bionic microstructure and smooth flat computing domain.

\subsection{RNG $k-\varepsilon$ model}

RNG k- $\varepsilon$ model is derived from the non-steady state NS equation and improved on the basis of the standard $k-\varepsilon$ model to make it more accurate and reliable in a wider range of flows. It performs well in simulating rotation in parallel flow and such strong swirl fluid flow. The equations of turbulent kinetic energy $k$ and turbulent dissipation $\varepsilon$ of the RNG $k-\varepsilon$ model are as follows:

$\frac{\partial}{\partial t}(\rho k)+\frac{\partial}{\partial x_{i}}\left(\rho k u_{i}\right)=\frac{\partial}{\partial x_{j}}\left[\alpha_{K} \mu_{e f f} \frac{\partial k}{\partial x_{j}}\right]+$

$G_{k}+G_{b}-\rho \varepsilon-Y_{M}+S_{K}$

$\frac{\partial}{\partial t}(\rho \varepsilon)+\frac{\partial}{\partial x_{i}}\left(\rho \varepsilon u_{i}\right)=\frac{\partial}{\partial x_{j}}\left[\alpha_{\varepsilon} \mu_{e f f} \frac{\partial \varepsilon}{\partial x_{j}}\right]+$

$C_{1 \varepsilon} \frac{\varepsilon}{k}\left(G_{k}+C_{3 \varepsilon} G_{b}\right)-C_{2 \varepsilon} \rho \frac{\varepsilon^{2}}{k}-R_{\varepsilon}+S_{\varepsilon}$

Where

$R_{\varepsilon}=C_{\mu} \rho \eta^{3}\left(1-\eta / \eta_{0}\right) \varepsilon^{3} /\left(\left(1+\beta \eta^{3}\right) k\right)$

$\eta \equiv S_{K} / \varepsilon$

$C_{1 \varepsilon}=1.44, C_{2 \varepsilon}=1.92, C_{3 \varepsilon}=0.09$

$\eta_{0}=4.38, \beta=0.012$

Here, $k$ is turbulent kinetic energy, $\varepsilon$ is turbulence dissipation, $u_{i}$ is component of velocity in $x_{i}$ 
direction, $\alpha_{k}$ is the inverse of the effective Prandtl number for turbulent kinetic energy $k, \alpha_{\varepsilon}$ is the inverse of the effective Prandtl number for urbulence dissipation $\varepsilon, G_{k}$ is turbulent kinetic energy term produced by laminar velocity gradient, $G_{b}$ is turbulent kinetic energy produced by buoyancy, $Y_{M}$ is contribution of fluctuation expansion to the total dissipation rate in compressible turbulence, $S_{k}$ is turbulent kinetic energy term defined by user, $S_{\varepsilon}$ is turbulent dissipation term defined by user, $\mu_{\text {eff }}$ is Equivalent dynamic viscosity coefficient.

\subsection{Meshing, boundary conditions and grid independence verification}

The mesh quality has a great influence on the convergence of computation and the accuracy of the final result. A high-quality structured meshing method is adopted for the calculation domain of the bionic microstructure surface in order to ensure the calculation accuracy. It is necessary to perform local mesh refinements in the near wall area and near the tip of the microstructure surface for focusing on the flow field in the boundary layer of the microstructure surface. The first layer of mesh thickness is estimated by the formula:

$y=\frac{y^{+} v}{u_{\tau}}$

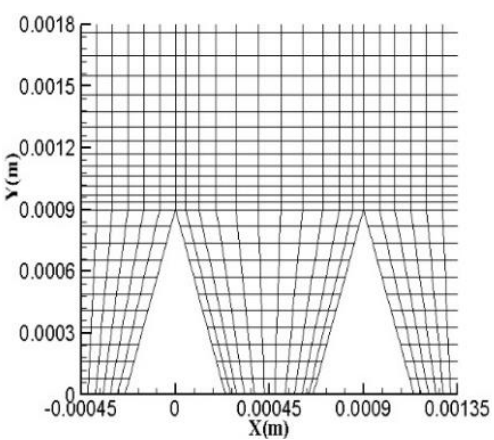

(a)

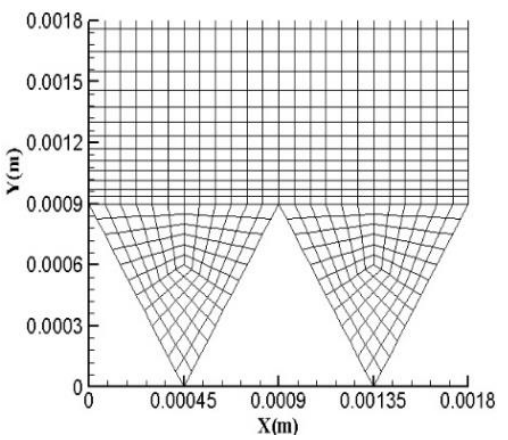

(b)

Fig. 3. Front view of the microstructure grid (a) V-shaped, (b) Serrated.

To ensure $y^{+}=1$, the thickness of the first layer of grid should be $4 \times 10^{-5}$. The vertical grid growth coefficient is set as 1.1. The front view and side view of two types of microstructure grid is shown in Fig. 3(a), Fig. 3(b) and Fig. 4.

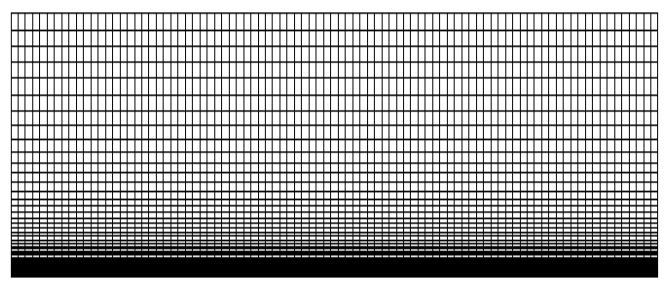

Fig. 4. Microstructure grid side view.

The entrance of the computational domain is set as the velocity entrance and the exit is set as the free flow exit. The two sides of the computational domain are set as periodic boundary conditions because the flow of the bionic microstructure surfaces in the span direction is periodic. The schematic diagram about boundary condition setting is shown in Fig. 5.

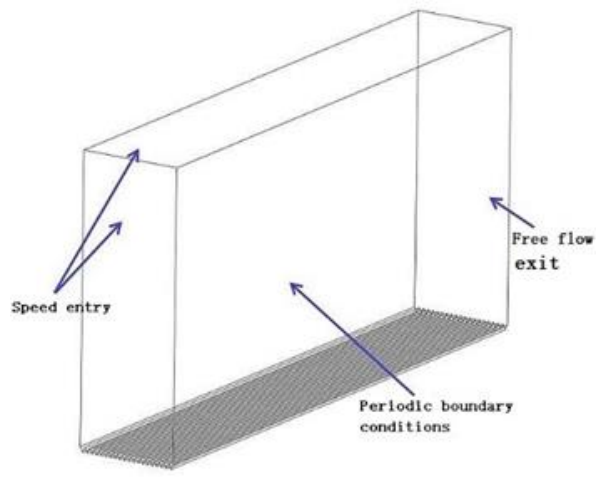

Fig. 5. Schematic diagram of boundary condition setting.

The RNG k- $\varepsilon$ model is used to carry out the numerical simulation analysis of the microstructure for drag reduction. The pressure-velocity-based solver is used and an incompressible ideal gas is selected for its incoming flow density. The viscosity was calculated by Sutherland method and the flow near the wall was treated with the enhanced wall function. The gradient difference method is based on Green - Gaussian node discrete method. The solution is solved using the SIMPLEC method. Pressure term, momentum term, turbulent kinetic energy term, dissipation term and energy term are discretized using the second order windward format. The relaxation factor is used default setting and the computational domain is carried out the Standard initialization.

The number of grids in the computational domain not only affects the accuracy of numerical simulation results, but also affects the duration of simulation process. In order to minimize the error caused by the number of grids and improve calculation efficiencies, Grid independence verification is carried out for the V-shaped bionic microstructure when $R e=2.53 \times 10^{6}$. The resistance coefficient is defined as follows: 


$$
C_{d}=\frac{F}{1 / 2 \rho U^{2} A}
$$

Where, $F$ is resistance on the surface of the microstructure, $A$ is the body surface area.

The resistance coefficient of the biomimetic microstructure is detected to observe whether the resistance coefficient changes with the number of grids. If the numerical simulation results are within the error tolerance range, it shows that the calculated values are independent of the number of meshes. The relationship between the resistance coefficient and the number of grids is shown in Tab. 2. It can be seen from the table that when the number of grids reaches 352,658 , the resistance coefficient tends to be stable basically. Therefore, it is more appropriate to divide the calculation domain of the $\mathrm{V}$-shaped bionic microstructure into 35,2658 grids, where the flow direction grid is set to 100 and the normal grid number is set to 80 . The same number of grids is used to the Serrated microstructures.

Table 2 Number of grids and resistance coefficient.

\begin{tabular}{|c|c|}
\hline \multicolumn{2}{|c|}{ coefficient. } \\
\hline Number of grids & $\frac{C_{d}}{10^{-5}}$ \\
\hline 143695 & 1.332 \\
\hline 180681 & 1.338 \\
\hline 253692 & 1.344 \\
\hline 306983 & 1.345 \\
\hline 352658 & 1.347 \\
\hline 473615 & 1.347 \\
\hline
\end{tabular}

\section{RESULTS AND DISCUSSION}

\subsection{Surface flow fields with different microstructures}

The biomimetic surface has a different flow field structure from the smooth plate surface. The differences of the flow field structure between the microstructure surface and the smooth plate surface are observed at the cross section $Z=0.02 \mathrm{~m}$ along the flow of the smooth plate and the biomimetic surface when the free flow velocity is at $5.5 \mathrm{~m} / \mathrm{s}$. The velocity contour of the smooth plate, the V-shaped microstructure and the Serrated microstructure at different sections along flow direction are shown in Fig. 6. The boundary layer on the microstructure surface is divided into the bottom and the top because of the existence of wall microstructure, which is different from the uniform distribution of boundary layer on the smooth plate surface. Moreover, the surface boundary layer above the microstructural valley floor is elevated, and the thickness of the linear bottom layer is increased. The fluid flow velocity in the near wall region of the bottom surface is also lower than that on the smooth plate surface. The velocity gradient in the boundary layer is reduced compared with the smooth plate. With turbulence fully developed, large amounts of "quiet" low-speed fluid begin to accumulate at the bottom of the microstructural valley.

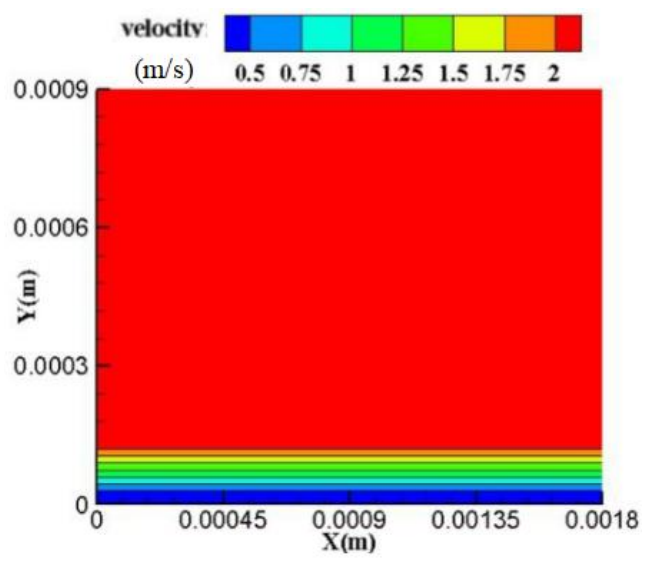

(a)

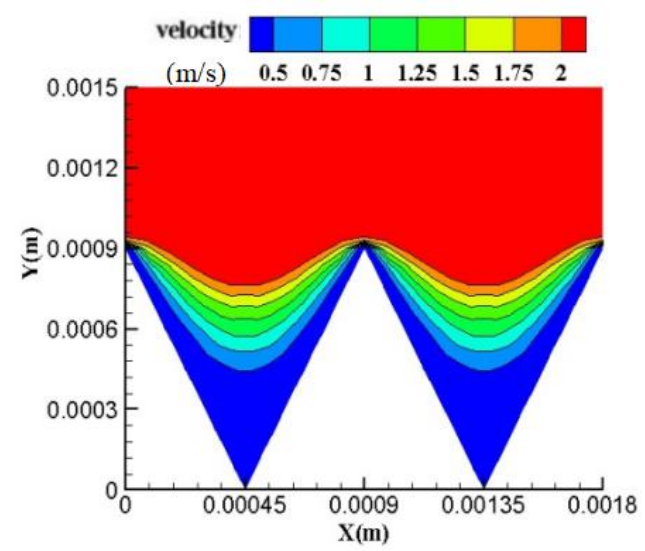

(b)

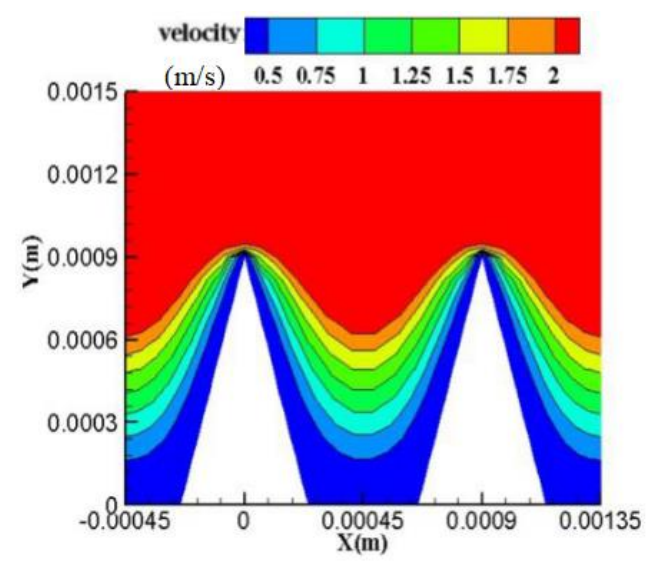

(c)

Fig. 6. velocity contour at different sections along flow direction (a) smooth plate, (b) V-shaped microstructure (c) Serrated microstructure.

The comparison of the drag coefficients between the two types of microstructures and the smooth plate with different flow speeds are shown in Fig. 7. It shows that the two bionic microstructures designed by the theoretical formulas can play a certain drag reduction effect within the range of flow speed, and the drag reduction performance between different types of microstructures is also different. In general, 
when the flow velocity is at $5-15 \mathrm{~m} / \mathrm{s}$, the drag reduction effect is the best.

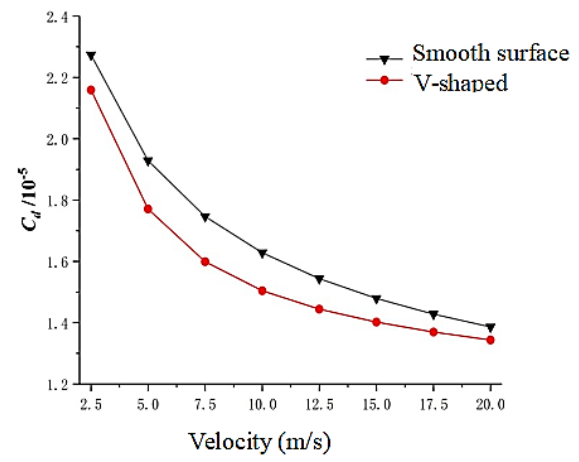

(a)

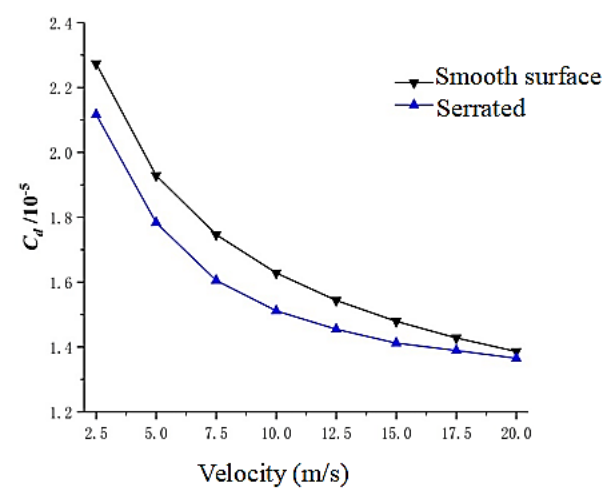

(b)

Fig. 7. Comparison of the drag coefficients between the microstructures and the smooth plate with different flow speeds (a) V-shaped (b) Serrated.

\subsection{Shear stress analysis of groove surface}

The biomimetic microstructures are placed downstream, so the pressure differential resistance of the microstructures can be negligible. The flow resistance is mainly provided by viscous shear stress, while the tangential wall stress can be expressed using velocity gradient as follows (Serrin 1959):

$$
\tau_{w}=\mu \frac{d u}{d y}
$$

The shear stress contour of the V-shaped microstructure and the serrated microstructure are shown in Fig. 8. The shear stress distribution on the surface of the microstructure is very uneven, and the high shear stress is mainly concentrated near the tip of the microstructure. The wall shear stress decreased greatly outside the tip areas of the microstructure and a large range of low wall shear stress exists near the bottom.

\subsection{Effects of parameters of microstructures on drag reduction}

In order to stud y the effects of microstructure parameters on the drag reduction, the drag coefficient of the solid wall of the bionic microstructure along the flow direction was monitored. When the drag coefficient remained stable and the residual curve reached $1 \mathrm{e}-07$, the simulation calculation was considered to converge and the calculation was stopped. The drag reduction rate of the microstructure is defined as follows:

$r_{f}=\frac{C_{d}-C_{d}{ }^{*}}{C_{d}} \times 100 \%$

Where, $\mathrm{Cd}$ is the resistance coefficient of the smooth plate, $C_{d}{ }^{*}$ is the resistance coefficient of the microstructure.

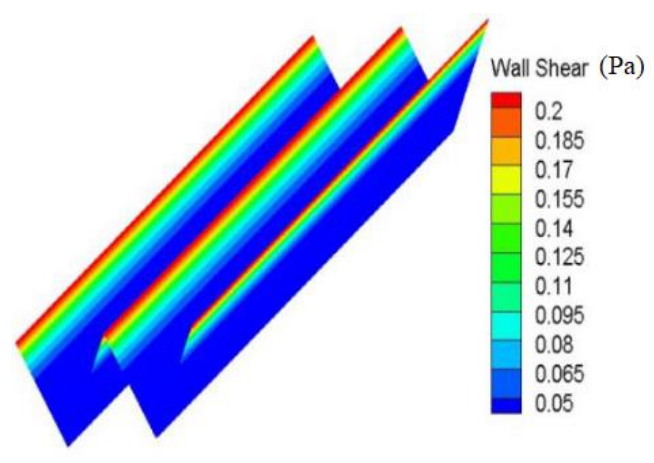

(a)

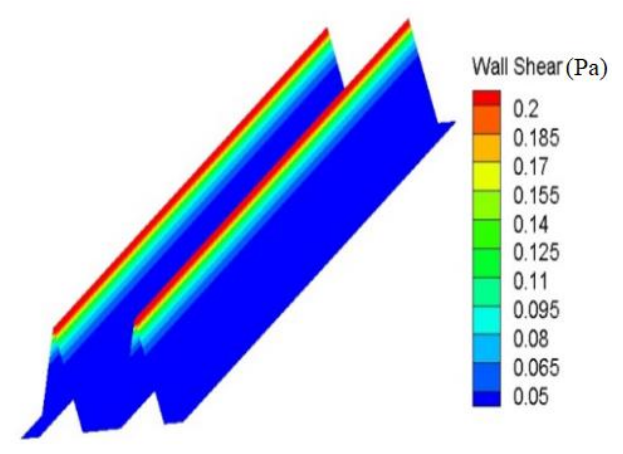

(b)

Fig. 8. Shear stress contour (a) V-shaped microstructure (b) Serrated microstructure.

\section{(1) Effects of microstructure height on drag reduction}

The distance between adjacent microstructures is kept constant and the height of the two types of microstructures is changed in order to discuss the influence of microstructure height on of biomimetic microstructure surface. Tab. 3 shows the size information of the two type of microstructures when the ratio of height to width is at $h / s=1.5, h / s=1.25$, $h / s=1, h / s=0.75, h / s=0.5$, and $h / s=0.25$.

The drag reduction effects of two types of microstructures with different $h / s$ (height/spacing) are numerically simulated when the velocity is at 7.5 $\mathrm{m} / \mathrm{s}$. Tab. 4 shows the drag reduction performance of two types of bionic microstructures at different $h / s$. 
M. H. Zhang et al. / JAFM, Vol. 14, No. 5, pp. 1337-1350, 2021.

Table 3 Size information of microstructures.

\begin{tabular}{|c|c|c|c|c|}
\hline \multirow{2}{*}{$h / s$} & Groove Types & Spacing $s(\mathrm{~mm})$ & Height $h(\mathrm{~mm})$ & Angle $\theta$ \\
\hline \multirow{2}{*}{1.5} & V-shaped & 0.9 & 1.35 & \\
\cline { 2 - 5 } & Serrated & 0.9 & 1.35 & $30^{\circ}$ \\
\hline \multirow{2}{*}{1.25} & V-shaped & 0.9 & 1.125 & \\
\cline { 2 - 5 } & Serrated & 0.9 & 1.125 & $30^{\circ}$ \\
\hline \multirow{2}{*}{1} & V-shaped & 0.9 & 0.9 & $30^{\circ}$ \\
\cline { 2 - 5 } & Serrated & 0.9 & 0.9 & $30^{\circ}$ \\
\hline \multirow{2}{*}{0.75} & V-shaped & 0.9 & 0.675 & $30^{\circ}$ \\
\cline { 2 - 5 } & Serrated & 0.9 & 0.675 & $30^{\circ}$ \\
\hline \multirow{2}{*}{0.5} & V-shaped & 0.9 & 0.45 & 0.45 \\
\cline { 2 - 5 } & Serrated & 0.9 & 0.225 & \\
\hline \multirow{2}{*}{0.25} & V-shaped & 0.9 & 0.225 & \\
\cline { 2 - 5 } & Serrated & 0.9 & & \\
\hline
\end{tabular}

Table 4 Drag reduction performance of microstructures.

\begin{tabular}{|c|c|c|c|c|c|}
\hline \multirow{2}{*}{$h / s$} & Groove Types & $\frac{C_{d}}{10^{-5}}$ & $s^{+}$ & $h^{+}$ & $r_{f}$ \\
\hline \multirow{2}{*}{1.5} & V-shaped & 1.604 & 15.07 & 22.60 & $8.1 \%$ \\
\cline { 2 - 5 } & Serrated & 1.645 & 15.08 & 22.62 & $5.75 \%$ \\
\hline \multirow{2}{*}{1.25} & V-shaped & 1.599 & 14.96 & 18.84 & $8.42 \%$ \\
\cline { 2 - 5 } & Serrated & 1.609 & 15.09 & 18.91 & $7.8 \%$ \\
\hline \multirow{2}{*}{1} & V-shaped & 1.598 & 15.22 & 15.22 & $8.43 \%$ \\
\cline { 2 - 5 } & Serrated & 1.602 & 15.18 & 15.18 & $8.2 \%$ \\
\hline \multirow{2}{*}{0.75} & V-shaped & 1.609 & 14.89 & 11.30 & $7.84 \%$ \\
\cline { 2 - 5 } & Serrated & 1.573 & 15.18 & 11.28 & $9.9 \%$ \\
\cline { 2 - 5 } & V-shaped & 1.627 & 15.04 & 7.53 & $6.8 \%$ \\
\hline \multirow{2}{*}{0.25} & Serrated & 1.571 & 14.95 & 7.49 & $10 \%$ \\
\cline { 2 - 5 } & V-shaped & 1.691 & 14.97 & 3.77 & $3.14 \%$ \\
\hline
\end{tabular}

It shows that the height changing of the microstructures has a very obvious effect on drag reduction. For different types of microstructures, the $h / s$, the dimensionless height $h^{+}$and spacing $s^{+}$are different when the drag reduction effects reach optimal drag reduction. For the V-shaped microstructure, the optimal drag reduction rate is up to $8.43 \%$ when $h / s=1$, its dimensionless height and spacing are $h^{+}=s^{+}=15.22$. However, for serrated microstructure, the optimal drag reduction rate is up to $10 \%$ when $h / s=0.5$, its dimensionless height and dimensionless spacing is at $h^{+}=7.49$ and $s^{+}=14.95$. In this manuscript, the grooves are set along the flow direction, which intercepts the movement of the near-wall fluid along the span direction and inhibits the momentum exchange between the fluid micelles, so that there are a large amount of low-speed and quiet fluid in the bottom area of the groove surface. The relative movement between the fluid cluster and the contact wall is small. So frictional resistance is low. However, secondary vortices are generated near the peak of the groove, and the friction at the top of the groove increases. This leads to a weakened drag reduction effect on the groove surface. For the $\mathrm{V}$-shaped groove, when $\mathrm{h} / \mathrm{s}$, for the serrated-shape, when $h / s=0.5$, there is relatively more space at the bottom of the groove to store low-speed fluid, the intensity of the secondary vortex is relatively low, so the frictional resistance is lower. This conclusion has been confirmed in many references (for example, Walsh 1983).

\section{(2) Effects of the tip angle on the drag reduction}

For a microstructure with a tip angle, the angle $\theta$ has a great influence on its drag reduction performance. In this part, we will discuss the effect on drag reduction with the change of microstructural tip angle. The tip angles are set to $\theta=20^{\circ}, 25^{\circ}, 30^{\circ}, 35^{\circ}$, $40^{\circ}$. The drag reduction rate of the five angles with different dimensionless dimensions is obtained by changing the incoming flow velocity. The dimensionless height of serrated microstructures at different dimensionless sizes are shown in Table 5 when the inflow velocity $U=5,7.5,10,12.5,15,17.5$ and $20 \mathrm{~m} / \mathrm{s}$.

The drag reduction effects between different angle serrated microstructures are shown in Fig. 9. It shows that the tip angle has a great effect on drag reduction for the serrated microstructures. The serrated microstructure surface with $\theta=40^{\circ}$ has a better drag reduction effect. Especially when the dimensionless height is at $h^{+}=13.79$, the drag reduction rate is up to $8.76 \%$. It also has relatively good drag reduction effect when the microstructure with the tip angle of $\theta=20^{\circ}$ and $35^{\circ}$. However, the microstructure with the tip angle $\theta=25^{\circ}$ has a poor drag reduction effect. 
M. H. Zhang et al. / JAFM, Vol. 14, No. 5, pp. 1337-1350, 2021.

Table 5 Drag reduction performance of serrated microstructures at different angles.

\begin{tabular}{|c|c|c|c|c|c|}
\hline \multirow{2}{*}{ Velocity (m/s) } & \multicolumn{5}{|c|}{$h^{+}$} \\
\cline { 2 - 6 } & $20^{\circ}$ & $25^{\circ}$ & $30^{\circ}$ & $35^{\circ}$ & $40^{\circ}$ \\
\hline 5 & 8.94 & 9.27 & 9.4 & 9.76 & 9.69 \\
\hline 7.5 & 12.93 & 13.19 & 13.38 & 13.62 & 13.79 \\
\hline 10 & 16.73 & 17.07 & 17.31 & 17.63 & 17.97 \\
\hline 12.5 & 20.57 & 20.96 & 21.23 & 21.62 & 21.85 \\
\hline 15 & 25.39 & 24.84 & 25.09 & 25.59 & 25.85 \\
\hline 17.5 & 28.22 & 28.71 & 29.04 & 29.55 & 29.84 \\
\hline 20 & 32 & 32.58 & 32.9 & 33.49 & 33.8 \\
\hline
\end{tabular}

According to the previous analysis, it shows that the wall shear stress in most areas of the microstructure is smaller than that of the smooth surface, especially at the bottom of the groove surface. Only near the top of the groove surface, the wall shear stress is slightly higher than that of the smooth surface. Compared with the smooth surface, the smaller the apex angle of the microstructure is, the more the wall shear stress of the microstructure decreases. However, it must be noted that the smaller the apex angle of the microstructure is, the larger the contact area between the surface of the structure and the flow field is. The drag reduction effect of the microstructure become weaker, and the total resistance may increase instead. Therefore, when the groove height $h$ is the same, there is an angle that maximizes the drag reduction efficiency. For the case in this manuscript, $\theta=40^{\circ}$.

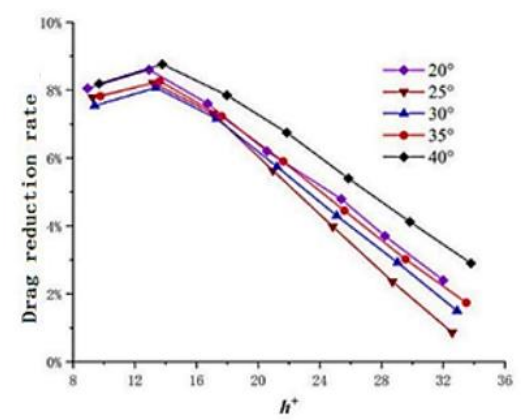

Fig. 9. Drag reduction rate of serrated microstructure at different tip angles.

The spanwise turbulent kinetic energy distribution are shown in Fig. 10 when the tip angle is at $\theta=40^{\circ}$ and $25^{\circ}$ of the serrated microstructure. It shows that the turbulent kinetic energy at tip angle $\theta=40^{\circ}$ is significantly less than that of $\theta=25^{\circ}$. The microstructure with the tip angle $\theta=40^{\circ}$ can suppress the pulsation of the nearby fluid better than the other tip angles so that the flow resistance is lower.

\subsection{Effect of wall temperature on surface boundary layer of microstructures}

As we know, transition process of smooth plate boundary layer can be delayed to the downstream as the wall temperature rises (Zhang et al. 2020). Thus, the laminar flow area is enlarged and the surface friction resistance is reduced. In this section, the effect on the resistance reduction was studied considering the combination of biomimetic surface and wall temperature control technology. The resistance reduction performance of the microstructure surface was numerically simulated with the change of the wall temperature. The resistance coefficients and drag reduction of the two types of microstructures are shown in Tab. 6 and Tab. 7. Let the temperature of the ambient flow $\mathrm{T}=288 \mathrm{~K}$. The wall temperature was selected to be $300 \mathrm{~K}$ and $450 \mathrm{~K}$ respectively. It shows that higher wall temperature can reduce the flow resistance on the surface of the microstructure and drag reduction effect has been significantly enhanced. In addition, the dimensionless height with the best drag reduction effect is also different with the changing wall temperature. For example, for V-shaped microstructure, the optimal drag reduction rate is up to $9.33 \%$ with the dimensionless height $h^{+}=13.47$ when wall temperature is at $T=300 \mathrm{~K}$. However, the optimal drag reduction rate is up to $12.6 \%$ with the dimensionless height $h^{+}=24.35$ when wall temperature is at $T=450 \mathrm{~K}$.

The thickness of the boundary layer increases with temperature. Without destroying the viscous bottom layer, there is relatively more space at the bottom of the groove to store low-speed fluid when the height of the groove is increased, the frictional resistance is lower. So, the optimal $h^{+}$increases as increasing wall temperatures.

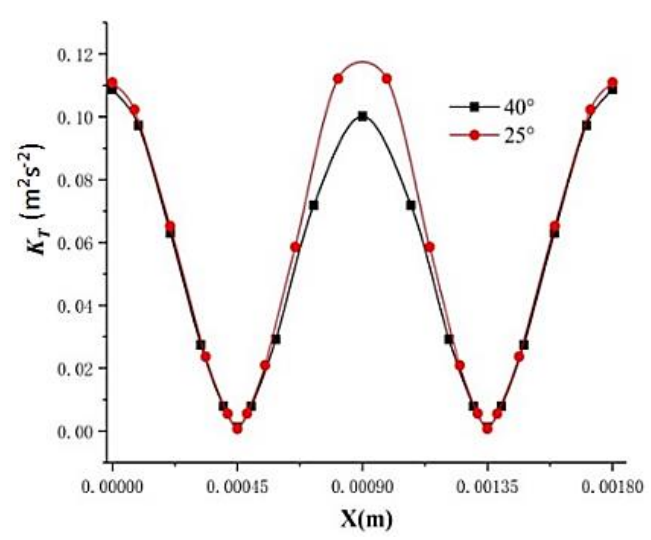

Fig. 10. Turbulent kinetic energy distribution of serrated microstructures at two angles. 
M. H. Zhang et al. / JAFM, Vol. 14, No. 5, pp. 1337-1350, 2021.

Table 6 Resistance reduction performance of microstructure at $T=300 \mathrm{~K}$.

\begin{tabular}{|c|c|c|c|c|}
\hline \multirow{2}{*}{ Velocity $(\mathrm{m} / \mathrm{s})$} & \multirow{3}{*}{ Groove Types } & \multicolumn{3}{|c|}{$T=300 \mathrm{~K}$} \\
\cline { 2 - 5 } & & $C_{d} / 10^{-5}$ & $h^{+}$ & $r_{f}$ \\
\hline \multirow{2}{*}{7.5} & V-shaped & 1.5832 & 13.47 & $9.33 \%$ \\
\cline { 2 - 5 } & Serrated & 1.6051 & 13.38 & $8.08 \%$ \\
\hline \multirow{2}{*}{10} & V-shaped & 1.4800 & 17.36 & $9.09 \%$ \\
\cline { 2 - 5 } & Serrated & 1.5113 & 17.31 & $7.17 \%$ \\
\hline \multirow{2}{*}{12.5} & V-shaped & 1.4155 & 21.23 & $8.29 \%$ \\
\cline { 2 - 5 } & Serrated & 1.4548 & 21.22 & $5.75 \%$ \\
\hline \multirow{2}{*}{15} & V-shaped & 1.3718 & 25.07 & $7.25 \%$ \\
\cline { 2 - 5 } & Serrated & 1.4120 & 25.09 & $4.54 \%$ \\
\hline \multirow{2}{*}{17.5} & V-shaped & 1.3411 & 28.66 & $6.08 \%$ \\
\cline { 2 - 5 } & Serrated & 1.3890 & 29.04 & $2.73 \%$ \\
\hline \multirow{2}{*}{20} & V-shaped & 1.3146 & 32.73 & $5.16 \%$ \\
\cline { 2 - 5 } & Serrated & 1.3651 & 32.90 & $1.52 \%$ \\
\hline
\end{tabular}

Table 7 Resistance reduction performance of microstructure at $T=450 \mathrm{~K}$.

\begin{tabular}{|c|c|c|c|c|}
\hline \multirow{2}{*}{ Velocity $(\mathrm{m} / \mathrm{s})$} & \multirow{3}{*}{ Groove Types } & \multicolumn{3}{|c|}{$T=450 \mathrm{~K}$} \\
\cline { 3 - 5 } & & $C_{d} / 10^{-5}$ & $h^{+}$ & $r_{f}$ \\
\hline \multirow{2}{*}{7.5} & V-shaped & 1.5679 & 13.41 & $10.21 \%$ \\
\cline { 2 - 5 } & Serrated & 1.5869 & 13.31 & $9.12 \%$ \\
\hline \multirow{2}{*}{10} & V-shaped & 1.4800 & 1.4446 & 17.16 \\
\cline { 2 - 5 } & Serrated & 1.5113 & 1.4585 & 17.01 \\
\hline \multirow{2}{*}{12.5} & V-shaped & 1.3555 & 20.78 & $12.18 \%$ \\
\cline { 2 - 5 } & Serrated & 1.3716 & 20.61 & $11.13 \%$ \\
\hline \multirow{2}{*}{15} & V-shaped & 1.2930 & 24.35 & $12.6 \%$ \\
\cline { 2 - 5 } & Serrated & 1.3185 & 24.25 & $10.85 \%$ \\
\hline \multirow{2}{*}{17.5} & V-shaped & 1.2498 & 28.03 & $12.47 \%$ \\
\cline { 2 - 5 } & Serrated & 1.2780 & 27.86 & $10.5 \%$ \\
\hline \multirow{2}{*}{20} & V-shaped & 1.216 & 31.47 & $12.27 \%$ \\
\cline { 2 - 5 } & Serrated & 1.2474 & 31.46 & $10 \%$ \\
\hline
\end{tabular}

Reynolds shear stress is the additional stress caused by the pulsation of fluid particles from the fluid layer with higher time-average velocity to the lower velocity layer, or generated the momentum exchange when the fluid particles pulsate from the low velocity layer to the high velocity layer. Reynolds shear stress is defined as follows:

$$
\tau^{R}=-\rho \overline{\left\langle u^{\prime} v^{\prime}\right\rangle}
$$

Noted that the author uses the Reynolds stress model to calculate the Reynolds stress in this manuscript since it is difficulty to obtaining pulsation velocity using RNG turbulence model.

Next, the influence of wall temperature on the boundary layer is discussed and the inflow velocity is set to $7.5 \mathrm{~m} / \mathrm{s}$.

The normal distribution of Reynolds shear stress of microstructures along the valley bottom in the boundary layer at different wall temperatures is shown in Fig. 11. It shows that the Reynolds shear stress in the linear bottom layer $\left(y^{+} \leq 10\right)$ decreases obviously when the wall temperature is up to $450 \mathrm{~K}$.
With the increase of the viscosity of the fluid in the boundary layer so that the momentum exchange between different layers in the boundary layer becomes weaker.

Figure 12 shows the turbulent kinetic energy in the boundary layer of two types of microstructures at wall temperature $T=300 \mathrm{~K}, 450 \mathrm{~K}$. The turbulent kinetic energy in the boundary layer is much lower when the wall temperature is at $450 \mathrm{~K}$. This indicates that the high wall temperature can suppress the pulsation of the boundary layer, that is, the fluid in the boundary layer became quieter with high temperature.

The fluid pulsation of the boundary layer is suppressed with the increase of wall temperature. The fluid in the microstructure boundary layer becomes quieter, and the momentum exchange between the layers in the boundary layer becomes weaker. And the Reynolds shear stress on the microstructure surface decreases significantly. As the temperature increases, the drag reduction effect is better. 


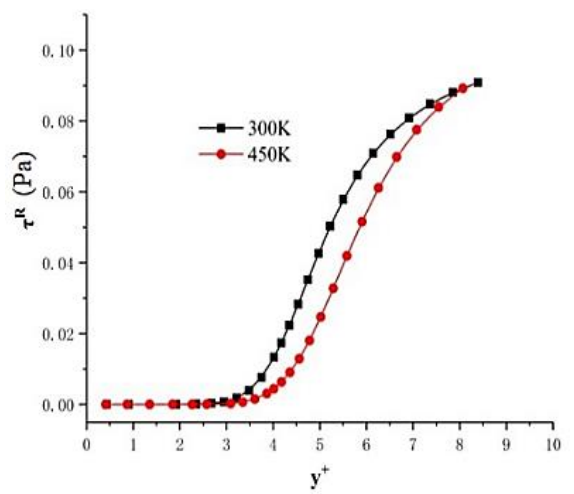

(a)

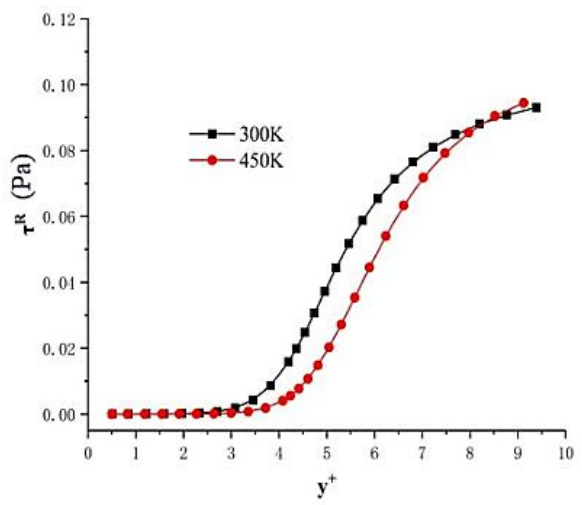

(b)

Fig. 11. Reynolds shear stress distribution in different wall temperatures (a) V-shaped microstructure (b) Serrated microstructure.

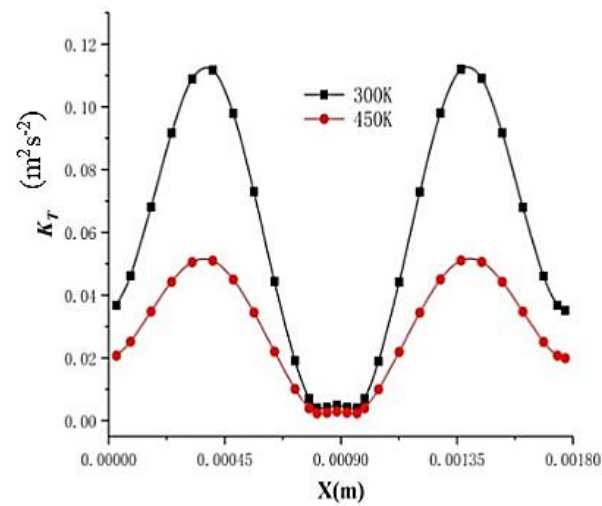

(a)

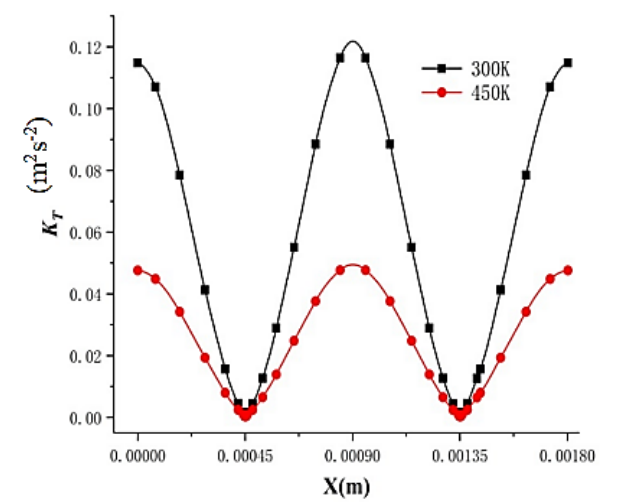

(b)

Fig. 12. Distribution of turbulent kinetic energy in different wall temperatures (a) V-shaped microstructure (b) Serrated microstructure.

\section{ANALYSIS OF DRAG REDUCTION} MECHANISM OF MICROSTRUCTURE

In this section, the internal velocity, vorticity, turbulence parameter, shear stress and flow evolution state of the microstructure were deeply explored from the boundary layer perspective.

Figure 13 shows the distribution of the Reynolds stress along the normal direction in the boundary layer between two types of microstructures and smooth plate surface. It shows that the Reynolds stress in the boundary layer of the microstructures is lower than that of the smooth flat surface. The existence of the microstructure weakens the momentum exchange in the boundary layer.

The vortex is a way to transfer energy during turbulent flow for the fluid. The smaller vorticity has the lower energy loss during the flow. Figure 14 shows the vorticity contour at the flow cross section $(\mathrm{Z}=1.8 \mathrm{~m})$ for the smooth plate and the two microstructures. It shows that the existence of the microstructure changes the vorticity distribution on the surface. The vorticity distribution is more uniform for the smooth flat surface. For the microstructures, the higher vorticity is concentrated near its tip while the vorticity in the turbulent boundary layer has been greatly weakened for the other parts of microstructures compared to the smooth flat surface.

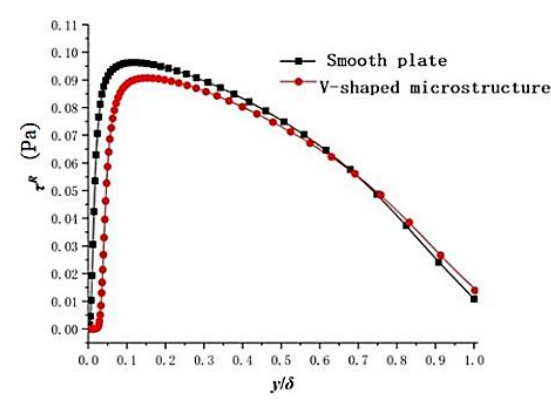

(a)

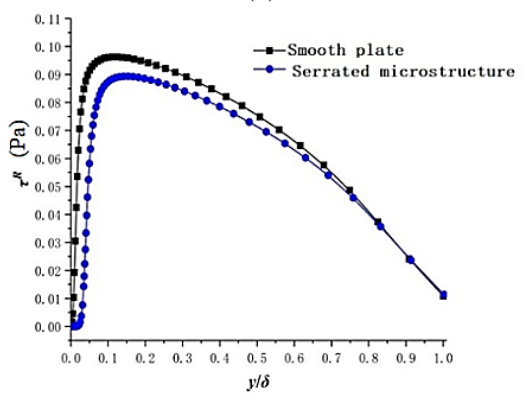

(b)

Fig. 13. Distribution of Reynolds stress along the normal direction in the boundary layer (a) V-shaped microstructure (b) Serrated microstructure. 
The existence of the wall microstructure inhibited the spreading vortex motion of the fluid, and the strength of the "counter-rotating vortex pair" was weakened, thus inhibiting the formation and development of the low-speed strip. The V-shaped microstructure has the lower vorticity value than that of the serrated microstructure. The vorticity arrangement is consistent with the drag reduction rates of the microstructures.

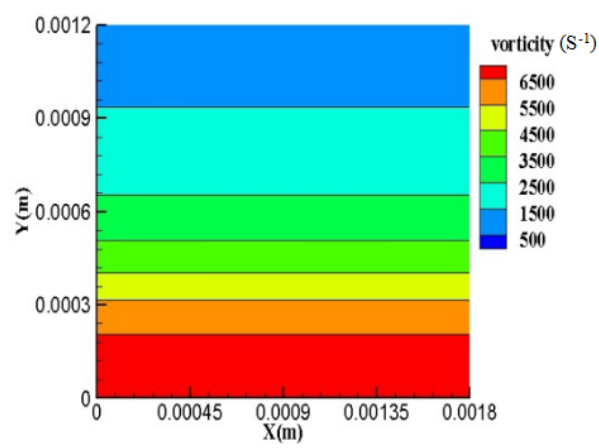

(a)

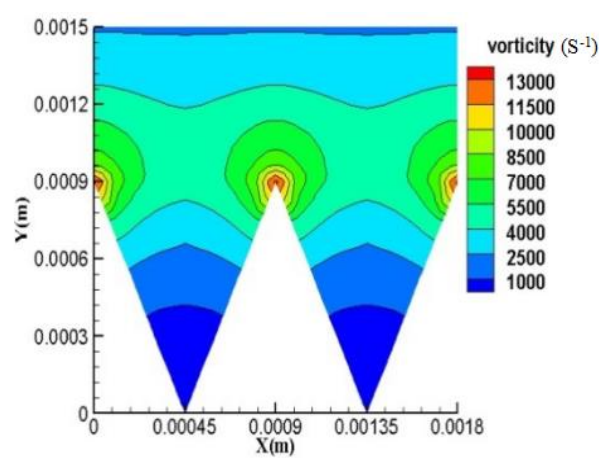

(b)

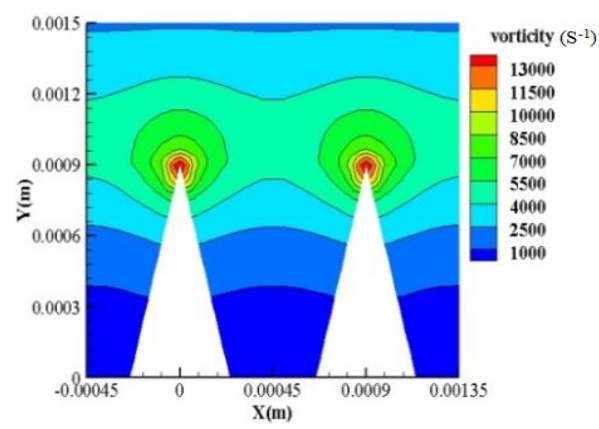

(c)

Fig. 14. Vorticity contour at the flow cross section $(Z=1.8 m)$ (a) smooth plate (b) $V$-shaped microstructure (c) Serrated microstructure.

Figure 15 shows that the deflection velocity contour of two types of microstructures in the span direction with the inflow velocity $U=7.5 \mathrm{~m} / \mathrm{s}$. The deflection velocity is caused by the deflection of the fluid near both sides of the tip when the fluid flows through the tip of the microstructure surface. It shows that the fluid is deflected near the tip as it flows through the surface of the microstructure.

Velocity vector of the microstructures are shown in Fig. 16. It shows that the velocity is deflected near

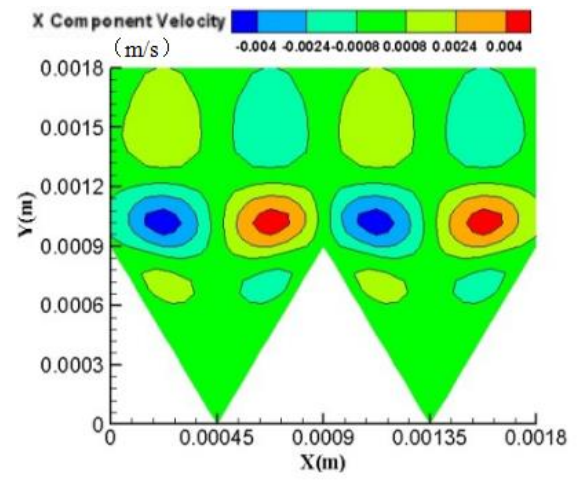

(a)

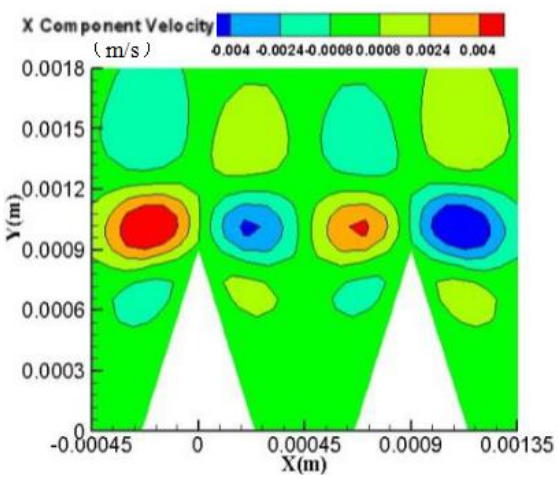

(b)

Fig. 15. Deflection velocity contour of the microstructures (a) V-shaped microstructure (b) Serrated microstructure.

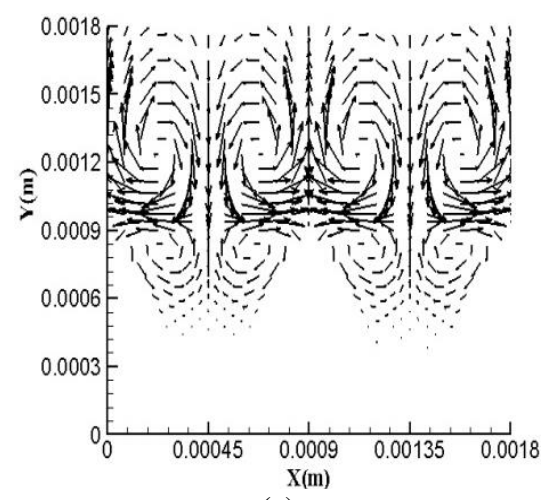

(a)

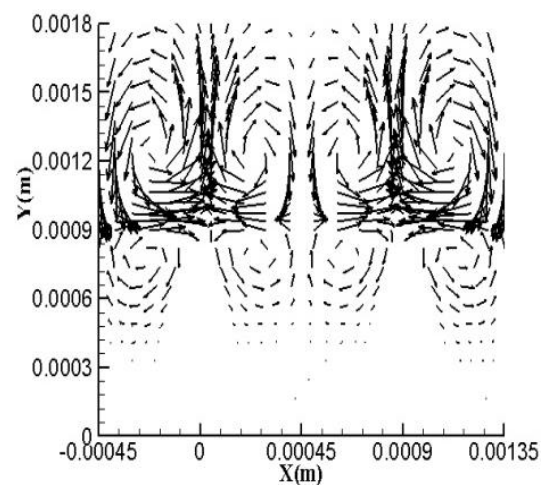

(b)

Fig. 16. Velocity vector of the microstructures (a) V-shaped microstructure (b) Serrated microstructure. 
the two sides of the tip when the fluid flows through the surface of the microstructures. And this deflection speed produces a pair of secondary vortices with opposite directions distributed on both sides of the microstructures. The formation of this special secondary vortex structure will restrict the movement of the fluid inside the boundary layer, which effectively limits the lateral pulsation of the fluid in the boundary layer, inhibits the upward lift of the low-speed band and weakens the intensity of the turbulent burst. At the same time, the existence of the secondary vortex pair causes a large amount of "quiet" fluid to gather at the valley bottom of the microstructures, which reduces the shear stress at the valley bottom, thereby reducing the flow resistance.

\section{Conclusion}

In this paper, the model of the $\mathrm{V}$-shaped and serrated bionic microstructures are established. The numerical simulation is carried out using the RNG $\mathrm{k}-\varepsilon$ model. The influence of microstructural dimension parameters on the drag reduction effect is discussed. Combined with wall temperature control of drag reduction technology, the influence of wall temperature on the drag reduction effect is analyzed. The drag reduction mechanisms of the bionic microstructures are explored from the boundary layer perspective. The key findings of this paper are listed as follows:

1 Under different dimensionless sizes, the Vshaped microstructure has the better drag reduction effect and the optimum drag reduction rate is up to $8.42 \%$. The drag reduction effect of microstructure is closely related to its height. The V-type microstructure reaches the best drag reduction rate when $h / s=1$ while for the serrated microstructure, can reach the best drag reduction rate when $h / s=0.5$ and the tip angle is at $\theta=40^{\circ}$.

2 When the surface temperature of the microstructure increases, the drag reduction effect is greatly enhanced. The dimensionless height (spacing) also changes to achieve the optimal drag reduction rate. for V-shaped microstructure, the optimal drag reduction rate is up to $9.33 \%$ with the dimensionless height

$h^{+}=13.47$ when wall temperature is at $T=300$ $\mathrm{K}$. However, the optimal drag reduction rate is up to $12.6 \%$ with the dimensionless height

$h^{+}=24.35$ when wall temperature is at $T=450 \mathrm{~K}$.

3 The existence of microstructure can weaken the momentum exchange in the boundary layer and inhibition the vortex motion of the fluid near the wall area. The secondary vortex generates in the opposite direction on both sides of the tip in the microstructures. The special secondary vortex structure can restrict transverse pulsation of the nearby fluid so that avoid the production of large eddy simulation and reduce the high speed fluid down sweep.
4 As the surface temperature rises, the thickness of the linear bottom layer can be increased. The flow state of the fluid near the wall becomes more stable. A large temperature gradient is formed in the boundary layer, which increases the viscosity of the fluid near the surface of the microstructure. The momentum exchange between the layers of the boundary layer is decreased to reduce the effect of flow resistance.

\section{REFERENCES}

Bechert, D. W., M. Bruse and W. Hage (2000). Experiments with three-dimensional riblets as an idealized model of shark skin. Experiments in Fluids 28(5), 403-412.

Chen, H., F. Rao, X. Shang, D. Zhang and I. Hagiwara (2013). Biomimetic drag reduction study on herringbone riblets of bird feather. Journal of Bionic Engineering 10(3), 341-349.

Dou, Z., J. Wang and D. Chen (2012). Bionic research on fish scales for drag reduction. Journal of Bionic Engineering 9(4), 457-464.

Domel, A. G., M. Saadat, J. C. Weaver, H.-H. Hossein, K. Bertoldi and G. V. Lauder (2018). Shark skin-inspired designs that improve aerodynamic performance. Journal of the Royal Society Interface 15, 20170828.

Feng, B., D. Chen, J. Wang and X. Yang (2015). Bionic Research on Bird Feather for Drag Reduction. Advances in Mechanical Engineering 7(2), 849294.

Fox, R. W., A. T. McDonald, P. J. Pritchard and J. C. Leylegia (2011). Introduction to Fluid Mechanics, John Wiley \& Sons, 8th edition, Chichester, UK 339-440

Fu, Y., C. Yuan and X. Bai (2017). Marine drag reduction of shark skin inspired riblet surfaces. Biosurface and Biotribology 3, 11-24.

Gregory, D. B. and B. Bhushan (2013). Fluid Drag Reduction with Shark-Skin Riblet Inspired Microstructured Surfaces. Advanced Functional Materials 23(36), 4507-4528.

Gu, Y. Q., T. X. Fan, J. G. Mou, D. H. Wu, S. H. Zheng and W. Evan (2017). Characteristics and mechanism investigation on drag reduction of oblique riblets. Journal of Central South University 24(6), 1379-1386.

Gu, Y. Q., S. W. Yu, J. G. Mou, T. X. Fan, S. H. Zheng and Z. Gang (2019). Experimental study of drag reduction characteristics related to the multifactor coupling of a bionic jet surface. Journal of Hydrodynamics 31(1), 186-194.

Liu, C., B. Chai, C. Liu and W. Ma (2015). Research 
on mechanism of drag reduction and efficiency improvement of hydrodynamic torque converter with bionic non-smooth surfaces. International Conference on Fluid Power and Mechatronics, Jilin University, Changchun, China.

Luo, J. and H. Zhou (1993). A theoretical model of the coherent structure in the wall region of a turbulent boundary layer. Applied Mathematics and Mechanics 14(11), 993-1001.

Luo, Y., Y. Liu, D. Zhang and E. Y. K. Ng (2014). influence of morphology for drag reduction effect of sharkskin surface. Journal of Mechanics in Medicine and Biology 14(02), 116.

Mariotti, A., G. Buresti and M.V. Salvetti (2014). Control of the turbulent flow in a plane diffuser through optimized contoured cavities. Eur. J. Mech. B Fluids 48, 254-265.

Mariotti, A., G. Buresti and M. V. Salvetti (2015). Use of multiple local recirculations to increase the efficiency in diffusers. European Journal of Mechanics - B/Fluids 50, 27-37.

Mariotti, A., G. Buresti, G. Gaggini and M. V. Salvetti (2017). Separation control and drag reduction for boat-tailed axisymmetric bodies through contoured transverse grooves. Journal of Fluid Mechanics 832, 514-549

Mariotti, A., G. Buresti and M. V. Salvetti (2019). Separation delay through contoured transverse grooves on a 2D boat-tailed bluff body: Effects on drag reduction and wake flow features. European Journal of Mechanics - B/Fluids 74, 351-362

Promode, R. B. and A. M. Hellum (2014). Modeling how shark and dolphin skin patterns control transitional wall-turbulence vorticity patterns using spatiotemporal phase reset mechanisms. Scientific Reports, 6650-6659.

Pu, X., Li, G. N. and H. Huang (2016). Preparation, anti-biofouling and drag-reduction properties of a biomimetic shark skin surface. Biology Open 5(4), 389-396.

Raayai-Ardakani, S. and G. McKinley (2017). Drag reduction using wrinkled surfaces in high Reynolds number laminar boundary layer flows. Phys. Fluids 29, 093605.

Rowley, C. W., T. Colonius and A. J. Basu (2002). On self-sustained oscillations in two- dimensional compressible flow over rectangular cavities. Journal of Fluid Mechanics 455, 315-346.

Schrader, L.-U (2019). Passive Drag Reduction via Bionic Hull Coatings. Journal of Ship Research 63(3), 206-218.

Serrin, J. (1959). Mathematical principles of classical fluid mechanics, in: S. Flügge(Ed.), Handbuch der Physik VIII/1, Springer-Verlag, Berlin, 125-263.

Song, X., M. Zhang and P. Lin (2017). Skin friction reduction characteristics of nonsmooth surfaces inspired by the shapes of Barchan Dunes. Mathematical Problems in Engineering 6212605, 1-10.

Walsh, M. J. (1983). Riblets as a viscous drag reduction technique. AIAA Journal 21(4), 485486.

Wang, X., X. Bai and C. Yuan (2015). Analysis of Present Development on Technologies of Antifouling and Drag Reduction Based on Bionic Non-smooth Surface. Ship Engineering 37.

Wu, L., Z. Jiao, Y. Song, C. Liu, H. Wang and Y. Yan (2018). Experimental investigations on drag-reduction characteristics of bionic surface with water-trapping microstructures of fish scales. Scientific Reports 8, 12186.

Wu, L., Z. Jiao, Y. Song, W. Ren, S. Niu and Z. Han (2017). Water-trapping and drag-reduction effects of fish Ctenopharyngodon idellus scales and their simulations. Science China Technological Sciences 60, 1111-1117.

Zhang, C. and K. S. Bijay (2018). Investigation on drag reduction performance of aero engine blade with micro-texture. Aerospace Science and Technology 72,380-396.

Zhang, M., W. Zhuo and G. Wang (2020). Study on the influence of wall temperature on the boundary layer of flat plate under multiparameters. Chinese Journal of Applied Mechanics 37, 755-760

Zhao, D. Y., Z. P. Huang, M. J. Wang, T. Wang and Y. Jin (2012). Vacuum casting replication of micro-riblets on shark skin for drag-reducing applications. Journal of Materials Processing Technology 212(1), 198-202. 